(C) 2022, The Authors. Published by Elsevier Inc. and Fass Inc. on behalf of the American Dairy Science Association ${ }^{\circledR}$. This is an open access article under the CC BY license (http://creativecommons.org/licenses/by/4.0/).

\title{
Quantitatively determining the somatic cell count of raw milk using dielectric spectra and support vector regression
}

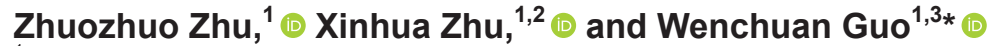 \\ ${ }^{1}$ College of Mechanical and Electronic Engineering, Northwest A\&F University, Yangling, Shaanxi, 712100, China \\ ${ }^{2}$ Shaanxi Research Center of Agricultural Equipment Engineering Technology, Yangling, Shaanxi, 712100, China \\ ${ }^{3}$ Key Laboratory of Agricultural Internet of Things, Ministry of Agriculture and Rural Affairs, Yangling, Shaanxi, 712100, China
}

\begin{abstract}
To investigate the potential of dielectric spectroscopy in quantitatively determining the somatic cell count (SCC) of raw milk, the dielectric spectra of 301 raw milk samples at different SCC were collected using coaxial probe technology in the frequency range of 20 to $4,500 \mathrm{MHz}$. Standard normal variate, Mahalanobis distance, and joint $\mathrm{x}-\mathrm{y}$ distances sample division were used to pretreat spectra, detect outliers, and divide samples, respectively. Principal component analysis and variable importance in projection (VIP) methods were used to reduce data dimension and select characteristic variables (CVR), respectively. The full spectra, 16 principal components obtained by principal component analysis, and 86 CVR selected by VIP were used as inputs, respectively, to establish different support vector regression models. The results showed that the nonlinear support vector regression models based on the full spectra and selected CVR using VIP had the best prediction performance, with the standard error of prediction and residual predictive deviation of $0.19 \mathrm{log}$ $\mathrm{SCC} / \mathrm{mL}$ and 2.37 , respectively. The study provided a novel method for online or in situ detection of the SCC of raw milk in production, processing, and consumption.
\end{abstract}

Key words: milk, somatic cell count, dielectric property, quantitative analysis

\section{INTRODUCTION}

Mastitis is an inflammatory response caused by udder infection of the dairy cow. It reduces milk production, degrades milk nutrients, and increases cost due to mastitis treatment (Halasa et al., 2007). For example,

Received June 6, 2021.

Accepted August 20, 2021.

*Corresponding author: wencguo@nwafu.edu.cn or guowenchuan69@ 126.com mastisitis causes the content of fat, casein, and lactose to reduce significantly, and the content of somatic cells, whey protein, fatty acids, sodium, chlorine, plasmin, and lipase to increase significantly (Ogola et al., 2007; Blowey and Edmondson, 2010). The monthly economic loss associated with dairy mastitis ranges from $\$ 12,000$ to $\$ 76,000$ in China (He et al., 2020). On the other hand, antibiotic residue in milk due to mastitis treatment has become a serious safety problem for human health. Therefore, detecting mastitis accurately and timely is important to reduce economic loss and ensure the quality and safety of dairy products.

There are various methods used in detecting dairy mastitis, including measuring the SCC (Dalen et al., 2019), electrical conductivity (Khatun et al., 2017), and viscosity (Kamphuis et al., 2008) of raw milk, or monitoring body temperature (Hovinen et al., 2008), rumination, and activity (Stangaferro et al., 2016) of cows. At present, SCC has been regarded as a gold standard for mastitis detection. Direct microscopy is a reference method for SCC detection, but it lacks specificity between cells and cytoplasmic particles, and its accuracy depends on operators. In practice, the developed automatic counters based on flow cytometry, such as Fossomatic 7 DC (Foss Electric) and Somacount FC (Bentley Instruments Inc.), are used to quickly and accurately measure the SCC of milk. However, high instrument prices and operation costs limit the use of these to some laboratories or large farms. In small farms, some low-cost tests, such as the California Mastitis Test, are widely used to measure SCC. However, the results are determined by observing sediment after reaction, causing highly subjective results (Ashraf and Imran, 2018). To develop rapid and online detection methods, near-infrared (NIR) spectroscopy has been used to measure the SCC of raw milk (Tsenkova et al., 2009; Iweka et al., 2020). The results indicate that the SCC could be predicted using NIR spectra based on the correlations between SCC and main components of milk. However, the fat globules in raw milk range from 1 to $20 \mu \mathrm{m}$ in size, causing serious scattering effect on 
NIR light. The size difference and unevenly distributed fat globules cause the obtained NIR spectra of raw milk to have low repeatability, limiting the detection precision on SCC by using NIR spectroscopy.

Dielectric properties describe the interaction of materials with the electromagnetic field, and they can enable fast and online detection with NIR spectroscopy. When mastitis occurs, a large number of somatic cells and electric ions present in raw milk are accompanied by a change of the overall milk composition. Therefore, the electric conductivity of milk has been used to detect the mastitis grades of dairy cows (Khatun et al., 2017), but the high false-positive rate makes it difficult for providing reliable results and cannot be used to quantitatively predict SCC. Several studies have shown that the dielectric properties of milk are closely related to its fat (Zhu et al., 2015b), protein (Zhu et al., 2015a), lactose (Liu et al., 2018), and salt (Zhu et al., 2018a) contents. Therefore, the dielectric spectra over a wide frequency range might provide more information related to SCC than the electric conductivity, and the dielectric spectra would be able to quantitatively detect the SCC of milk, but the potential has not been investigated. Therefore, the objectives of this research were (1) to collect the dielectric spectra of raw milk at different SCC in the frequency range of 20 to $4,500 \mathrm{MHz},(2)$ to establish quantitative models for predicting the SCC of raw milk, and (3) to provide the theoretical basis for achieving rapid and online detection of SCC based on the dielectric spectra of raw milk.

\section{MATERIALS AND METHODS}

\section{Milk Samples}

In this study, the raw milk samples were obtained from 3 local farms in Yangling, Shaanxi. When 1 or 2 suspected cases of clinical mastitis were found by feeders, the milk samples (mastitis samples) from the suspected cases were collected. Meanwhile, 9 milk samples (normal samples) were obtained from cows without clinical mastitis features. Each milk sample was collected from 4 udder quarters of a single cow, and the first 3 handfuls of milk were discarded. The collected samples were transported from farms to the laboratory within 20 min.

To obtain more samples at different SCC, the mastitis sample was mixed with randomly selected normal samples at the ratios of $2: 1,1: 2,1: 6$, and $1: 12$ when 1 mastitis sample was collected. The experiment was conducted 7 times, and a total of 7 original mastitis samples, 63 normal samples, and 28 mixed samples using mastitis and normal samples were obtained.
When 2 mastitis samples were collected, the 2 original mastitis samples were mixed at the ratios of $4: 1$ and 1:4 to obtain 2 mixed mastitis samples. Then, each original or mixed mastitis sample was mixed with randomly selected normal samples at the ratios of $2: 1,1: 2$, $1: 6$, and 1:12. The experiment was carried out 7 times, and 14 original mastitis samples, 63 normal samples, and 126 mixed samples (14 mixed mastitis samples and 112 mixed samples using mastitis and normal samples) were obtained.

In total, 301 raw milk samples were obtained, including 21 original mastitis samples, 126 normal samples, and 154 mixed samples. Each prepared sample was stirred for 1 min using a stirring electric heating mantle (CL1, Shanghai Lichen Bangxi Instrument Technology Co., Ltd.) and divided into 2 parts. We added $0.6 \mathrm{~g} / \mathrm{L}$ potassium dichromate (Tianjin Bodi Chemical Co., Ltd., analytical grade, $99.8 \%$ ) to one part and stored it in a $4^{\circ} \mathrm{C}$ refrigerator for the analysis of SCC and main components within $48 \mathrm{~h}$ after milking. Another part was used to measure the dielectric spectra, electric conductivity, and $\mathrm{pH}$ at room temperature $\left(24-26^{\circ} \mathrm{C}\right)$ within $6 \mathrm{~h}$ after milking. To avoid milk deterioration, these milk samples were also kept in a $4^{\circ} \mathrm{C}$ refrigerator before measurements. All samples had no deterioration during experiments.

\section{Measurement on Milk Components and SCC}

The SCC and main milk components (fat, protein, lactose, and total solids contents) were measured using a milk analyzer (Combi 500, Bentley Instruments Inc.) in the Dairy Herd Improvement Center of Shaanxi Provincial Animal Husbandry Technology Extension Station. The technology used to detect SCC was flow cytometry. The relative mean difference of the milk analyzer for SCC was below $10 \%$, and it met the standard 13366-2 of the International Organization of Standardization (ISO, 2006). Sample analysis was conducted in triplicate, and the mean was used as the result. Owing to the very wide change range of SCC, the logarithmic SCC at base 10 was used for further quantitative analyses.

\section{Measurement on $\mathrm{pH}$ and Electrical Conductivity}

A pH meter (PHSJ-3F, Shanghai Precision \& Scientific Instrument Co., Ltd.) and a conductivity meter (DDSJ-308A, Shanghai Precision \& Scientific Instrument Co., Ltd.) were used to measure the $\mathrm{pH}$ and electrical conductivity of each milk sample at room temperature $\left(25^{\circ} \mathrm{C}\right)$, respectively. The mean of triplicate was reported in this study. 


\section{Measurement on Dielectric Spectra}

The dielectric spectra of milk samples were collected using a dielectric properties measurement system based on open-ended coaxial probe technology. The system was mainly composed of an Agilent E5071C vector network analyzer (Agilent Technologies), an Agilent 85070E coaxial probe, an Agilent 85070 test software, a water bath (KD-98-1, Tianjin Test Instrument Co., Ltd.), a hydraulic lifting platform, and a computer. After the system was warmed up for about $1 \mathrm{~h}$, the vector network analyzer and coaxial probe were calibrated. The detailed information and calibration methods of the system were described elsewhere (Guo et al., 2010).

Each sample was poured into three $10-\mathrm{mL}$ beakers with an inner diameter of $22.5 \mathrm{~mm}$ and a depth of 25 $\mathrm{mm}$. The beaker filled with milk was placed in the water bath that was set to $25^{\circ} \mathrm{C}$. A thermometer was used to check the sample temperature. When the sample temperature reached and remained at $25^{\circ} \mathrm{C}$, the coaxial probe was immersed into the milk sample by elevating the water bath with the hydraulic lifting platform. To make the spectra have enough data related to the SCC of milk and to reduce the time used in data processing, modeling, and prediction, based on our experiences and other studies (Guo et al., 2010; Zhang et al., 2016), the dielectric spectra (dielectric constant $\varepsilon^{\prime}$ and dielectric loss factor $\varepsilon^{\prime \prime}$ ) of the milk sample were obtained at 201 discrete frequencies from 20 to $4,500 \mathrm{MHz}$. The measurement was repeated 3 times for the milk in each beaker, and the mean of 9 measurements was used as the final result for each sample.

\section{Data Processing}

Data processing, including spectra pretreatment, outlier detection, and sample division, is helpful to establish a stable and accurate quantitative model. In this study, Savitzky Golay (SG) smoothing, standard normal variate $(\mathbf{S N V})$, and their combination $(\mathbf{S G}+\mathbf{S N V})$ were used to pretreat the obtained dielectric spectra. The SG smoothing and SNV could eliminate high-frequency noises and separate the physical and chemical information in spectral data, respectively. The details about SG smoothing and SNV could be found in other studies (Zhu et al., 2018b).

The Mahalanobis distance (MD) can be used for automatically locating the spectra that are far from the center of the data set and is suitable for outlier detection (Mahalanobis, 1936). Because the high correlations between variables at different frequencies cause the variance-covariance matrix used in MD be too ill-conditioned for inversion, the first few principal components (PC) obtained by principal component analysis (PCA) with the accumulative contribution rate greater than $99.9 \%$ were used to calculate the MD of each sample. For samples where MD exceeded 3 times standard deviations of the mean MD of all samples, we considered them as outliers.

After the outliers were removed, the joint $\mathrm{x}-\mathrm{y}$ distances (SPXY; Galvão et al., 2005) method was used to divide the samples into the calibration and prediction sets at the ratios of $2: 1$ and $3: 1$. The optimal pretreatment method and sample division ratio were determined according to the prediction performance of the established linear support vector regression (LSVR) models.

\section{Data Reduction}

Dimension reduction is usually used to map the highdimensional original variables space to a low-dimensional space for reducing collinearity and improving the signal-to-noise ratio of the data. Variable selection is helpful to improve the model prediction performance by removing noninformative or interferential variables. In this study, dimension reduction and variable selection were conducted to compress the pretreated dielectric spectra.

Partial least squares (PLS) and PCA are widely used to map the original high-dimensional original variables to low-dimensional latent variables $(\mathbf{L V})$ and $\mathrm{PC}$, respectively. The LV are sorted according to their relevance to the $y$ variables (SCC here), and PC are sorted according to the explained variance of the spectra matrix (dielectric spectra here; Nicolaï et al., 2007). The optimal numbers of $\mathrm{LV}$ and $\mathrm{PC}$ after dimension reduction were decided by the prediction performances of built LSVR models with different numbers of LV and PC.

Variable importance in projection (VIP) and Monte Carlo uninformative variable elimination (MC-UVE) methods, which are based on the parameters of PLS regression (PLSR) model, were used to select characteristic variables (CVR) in this study. The optimal PLSR model was determined by using the leave-one-out cross-validation method and root-mean-squares error of cross-validation (RMSECV). When the CVR were chosen using the VIP method, the whole calibration set was used to build the optimal PLSR model. The VIP values, the weighted average sum of the weights in the built PLSR model, were calculated to evaluate the importance of the dielectric variables (Farrés et al., 2015). Because the average square of VIP values is 1 , the dielectric variable was selected as the CVR if its VIP value was greater than 1 .

When the CVR were selected by using MC-UVE, 500 Monte Carlo samplings were performed for the calibration set. Eighty percent of the total samples were 
selected at each time, and then the optimal PLSR models were constructed to obtain the regression coefficient matrixes. Two hundred random variables were added to the pretreated dielectric spectra before sampling, and the stability of the dielectric variables and random variables were both calculated (Cai et al., 2008). The stability greater than $95 \%$ of the random variable was regarded as the threshold in this study.

\section{Modeling Methods}

Support vector regression (SVR), a learning machine based on statistical learning theory and structural risk minimization assumptions, is usually used to minimize the empirical risk and confidence interval and to improve generalization ability. For the LSVR model, the estimation function $F(\mathrm{X})$ is as follows:

$$
F(\mathrm{X})=\mathbf{W X}+\mathbf{b}
$$

where $\mathbf{X}$ is the input matrix, $\mathbf{W}$ is the regression coefficient matrix, and $\mathbf{b}$ is the threshold.

For nonlinear problems, nonlinear SVR (NSVR) uses the kernel function $\Phi(\mathrm{X})$ to map the input matrix $\mathbf{X}$ to a high-dimensional feature space. The estimation function of NSVR is to replace $\mathbf{X}$ in Eq. [1] with $\Phi(\mathrm{X})$. The regression coefficient matrix $\mathbf{W}$ could be obtained by minimizing Eq. [2] as follows:

$$
\frac{1}{2}\|\mathbf{W}\|^{2}+C \frac{1}{n} \sum_{i=1}^{n} \max \left\{0,\left|y_{i}-f\left(x_{i}\right)\right|-\zeta\right\},
$$

where $C$ is the regularization parameter to balance training error and model complexity, $n$ is the number of samples, $y_{\mathrm{i}}$ is the reference value of the $i$ th sample, $f\left(x_{\mathrm{i}}\right)$ is the predicted value of $i$ th sample, and $\zeta$ is the maximum tolerant error of the model.

Equation [2] indicates that the sample with a predicted error greater than $\zeta$, named as support vector, has an effect on model optimization. Therefore, $\zeta$ indirectly affects the support vector ratio and controls the model's sparsity.

Because the accuracy of $f\left(x_{\mathrm{i}}\right)$ is unknown, optimizing $\zeta$ is difficult. By default, $\zeta$ was set to 0.1 in the LSVR and NSVR models in this study. Schölkopf et al. (2000) modified the SVR algorithm and introduced a parameter $v$ to approximate the training error and support vector ratio, which is called $\mathrm{Nu}$-Support vector regression (NuSVR). Similar to the SVR algorithm, the kernel function $\Phi(\mathrm{X})$ can also be used to construct a nonlinear NuSVR (NNuSVR) for nonlinear problems.

Various nonlinear kernel functions, including polynomials, sigmoid, and radial basis functions (RBF), are developed for SVR models. Because RBF can simplify the model training process and provide good prediction performance (Zhu et al., 2018b), it was used as the kernel functions of NSVR and NNuSVR models here.

In this study, LSVR, NSVR, and NNuSVR models were established to predict the SCC of raw milk. By comparing the prediction results of the 3 linear or nonlinear SVR models, the potential of dielectric spectra in predicting the SCC of milk was investigated.

\section{Model Performance Assessment}

The RMSECV was used to select the optimal parameters in different models. The correlation coefficient (r) and standard error of prediction (SEP) were calculated to evaluate the prediction performance of the model for calibration ( $\mathbf{r}_{\mathbf{c}}$ and $\left.\mathbf{S E P C}\right)$ and prediction $\left(\mathbf{r}_{\mathrm{p}}\right.$ and SEPP) sets. The residual prediction deviation (RPD) was also used to evaluate the prediction ability of the model. The definitions of these parameters could be found elsewhere (Nicolaï et al., 2007).

\section{Software}

In addition to the 85070D software used in dielectric spectra collection, SPSS 17.0 (SPSS Inc.) was applied to perform statistic analysis on the main components, $\mathrm{SCC}, \mathrm{pH}$, and electric conductivity of milk samples, and to analyze the correlations of SCC with the main components, $\mathrm{pH}$, electric conductivity, and dielectric properties at a given frequency. Data processing and reduction were conducted in Matlab 2010 (The Mathworks Inc.). The LSVR, NSVR, and NNuSVR models were established using Spyder 3.2.6 (https://www .spyder-ide.org) based on the Scikit-Learn machine learning package (Pedregosa et al., 2011).

\section{RESULTS AND DISCUSSION}

\section{Statistic Results of Used Milk Samples}

Table 1 shows the statistic results on SCC, main components, $\mathrm{pH}$, and electric conductivity of the used 301 raw milk samples, and the correlation analysis results of SCC with main components, $\mathrm{pH}$, and electric conductivity. The SCC ranged from $3.70 \log \mathrm{SCC} / \mathrm{mL}$ to $6.70 \log \mathrm{SCC} / \mathrm{mL}$, with the mean value and standard deviation of $5.78 \log \mathrm{SCC} / \mathrm{mL}$ and $0.60 \log \mathrm{SCC} /$ $\mathrm{mL}$, respectively. The fat, protein, lactose, and total solids contents of milk samples were $3.34 \pm 0.81 \%, 3.14$ $\pm 0.39 \%, 4.91 \pm 0.23 \%$, and $11.77 \pm 1.15 \%$ (mean \pm $\mathrm{SD})$, respectively. The $\mathrm{pH}$ and electric conductivity of milk samples were $6.64 \pm 0.64$ and $4.12 \pm 0.73 \mathrm{mS} / \mathrm{cm}$, respectively. The results showed that the SCC, main 
Table 1. Statistical results on SCC, main compositions, $\mathrm{pH}$, and electrical conductivity of milk samples and correlation analysis (r) of SCC with the main components, $\mathrm{pH}$, and electrical conductivity (EC)

\begin{tabular}{lccccccc}
\hline Parameter & SCC $(\log \mathrm{SCC} / \mathrm{mL})$ & Fat $(\%)$ & Protein $(\%)$ & Lactose $(\%)$ & TS $(\%)$ & $\mathrm{pH}$ & EC $(\mathrm{mS} / \mathrm{cm})$ \\
\hline Minimum & 3.70 & 1.35 & 2.36 & 3.62 & 8.63 & 6.34 & 2.09 \\
Maximum & 6.70 & 5.55 & 4.66 & 5.43 & 14.87 & 7.20 \\
Mean & 5.78 & 3.34 & 3.14 & 4.91 & 11.77 & 6.64 & 4.02 \\
SD & 0.60 & 0.81 & 0.39 & 0.23 & 1.15 & 0.64 & 0.73 \\
$\mathrm{r}$ & -1 & -0.075 & 0.051 & $0.583^{*}$ & -0.078 & 0.051 \\
\hline
\end{tabular}

${ }^{1}$ Analysis was not done.

$* P<0.05$.

components, $\mathrm{pH}$, and electrical conductivity of milk samples from cows in different dairy farms, lactation stages, and mastitis health status varied widely, which indicated that the samples used in this study were representative. The correlations between the SCC and fat, protein, total solids, and $\mathrm{pH}$ were not significant $(P>$ $0.05)$, and the absolute values of $\mathrm{r}$ were less than 0.1 . However, SCC was significantly correlated with lactose and electrical conductivity $(P \leq 0.05)$ with r-values of 0.583 and 0.524 , respectively.

\section{Dielectric Properties of Milk Samples}

Figure 1a shows the original $\varepsilon^{\prime}$ spectra of $301 \mathrm{milk}$ samples over the frequency range of 20 to $4,500 \mathrm{MHz}$. It shows that the $\varepsilon^{\prime}$ decreased with increasing frequency over the whole frequency range. The decreased $\varepsilon^{\prime}$ with the increased frequency was more obvious below 40 $\mathrm{MHz}$. The $\varepsilon^{\prime}$ characterizes the performance of materials in storing energy, which is mainly caused by the rotation of dipoles with the direction change of electric field. When the frequency increases, a part of dipoles will not be able to follow the direction change of electric field, causing a decrease in $\varepsilon^{\prime}$. The effect of frequency on the $\varepsilon^{\prime}$ of milk was also reported in other studies (Zhu et al., 2015a).

Figure $1 \mathrm{~b}$ shows the original $\varepsilon^{\prime \prime}$ spectra of the total milk samples at frequencies between $20 \mathrm{MHz}$ and 4,500 $\mathrm{MHz}$. When the frequency was lower than 1,000 MHz, the $\varepsilon^{\prime \prime}$ decreased linearly with increasing frequency on a logarithmic scale. When the frequency was greater than $1,800 \mathrm{MHz}, \varepsilon^{\prime \prime}$ increased with the increase of frequency. The $\varepsilon^{\prime \prime}$ is related to the energy loss of material. At low frequencies, the movement of positive and negative ions in milk due to the direction change of electric field leads to energy loss. This phenomenon becomes weak with increasing frequency, resulting in the decrease of $\varepsilon^{\prime \prime}$ with increasing frequency. As the frequency increases continuously, the dipoles in the milk can no longer follow the direction change of electric field, which increases the energy loss and $\varepsilon^{\prime \prime}$. The same trend has also been found in other studies (Zhu et al., 2018a).

\section{Linear Correlation Between Dielectric Properties and SCC}

A large number of somatic cells enter raw milk because of dairy mastitis, which could increase the $\varepsilon^{\prime}$ (Patel et al., 2008; Xiong et al., 2008). Meanwhile, the electric ions related to $\varepsilon^{\prime \prime}$ increase in milk. The linear correlations between the dielectric properties $\left(\varepsilon^{\prime}\right.$ and $\left.\varepsilon^{\prime \prime}\right)$ at 201 frequencies with SCC are shown in Figure 2. At the frequency below $100 \mathrm{MHz}$, the $\mathrm{r}$ between $\varepsilon^{\prime}$ and SCC $\left(\mathrm{r}_{\varepsilon^{\prime}}\right)$ dropped from about 0.4 to about -0.05 with the increase of frequency, and changed between 0 and -0.1 above $100 \mathrm{MHz}$. For $\varepsilon^{\prime \prime}$, when the frequency was lower than 1,000 MHz, the $\mathrm{r}$ between $\varepsilon^{\prime \prime}$ and $\mathrm{SCC}\left(\mathbf{r}_{\varepsilon^{\prime \prime}}\right)$ changed between 0.5 and 0.6 ; in particular, for frequencies below $\sim 300 \mathrm{MHz}, \mathrm{r}_{\varepsilon^{\prime \prime}}$ was maintained close to 0.55 . The $r_{\varepsilon^{\prime \prime}}$ decreased rapidly with increasing frequency above 1,000 MHz. Generally, at a given frequency, $\varepsilon^{\prime \prime}$ had a better linear correlation with SCC than $\varepsilon^{\prime}$, but the SCC cannot be predicted accurately because of the low correlation. Therefore, it is necessary to explore the feasibility of predicting SCC accurately by applying the dielectric spectra over a wide frequency range.

\section{Data Processing}

The milk samples were numbered from 1 to 301. The MD was used to detect outliers based on the original spectra and the pretreated spectra using SG smoothing, SNV, and SG+SNV. The sample numbers of the identified outliers are listed in Table 2. It can be seen that the pretreatment methods affected the detected outliers.

According to the ratios of $2: 1$ and $3: 1$, the samples after removing outliers were divided by the SPXY method, and then the LSVR models were established. Table 2 shows the prediction results of the built LSVR models in the calibration and prediction sets. It indicates that after removing the identified 5 outliers based on the pretreated spectra using SNV, the established LSVR model at the sample division ratio of 3:1 had the smallest SEPP of $0.22 \log \mathrm{SCC} / \mathrm{mL}$. Therefore, the SNV was 
Table 2. The prediction results of linear support vector regression models at different spectral pretreating methods and sample division ratios with the Mahalanobis distance as the outlier detection method

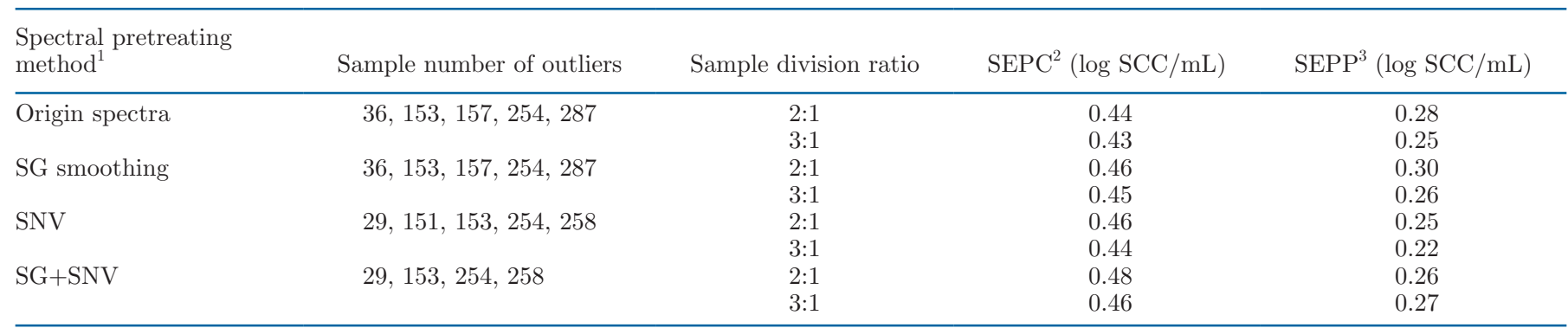

${ }^{1} \mathrm{SG}=$ Savitzky Golay $; \mathrm{SNV}=$ standard normal variate $; \mathrm{SG}+\mathrm{SNV}=$ combination of $\mathrm{SG}$ and SNV.

${ }^{2} \mathrm{SEPC}=$ standard error of prediction for calibration.

${ }^{3} \mathrm{SEPP}=$ standard error of prediction for prediction.

A
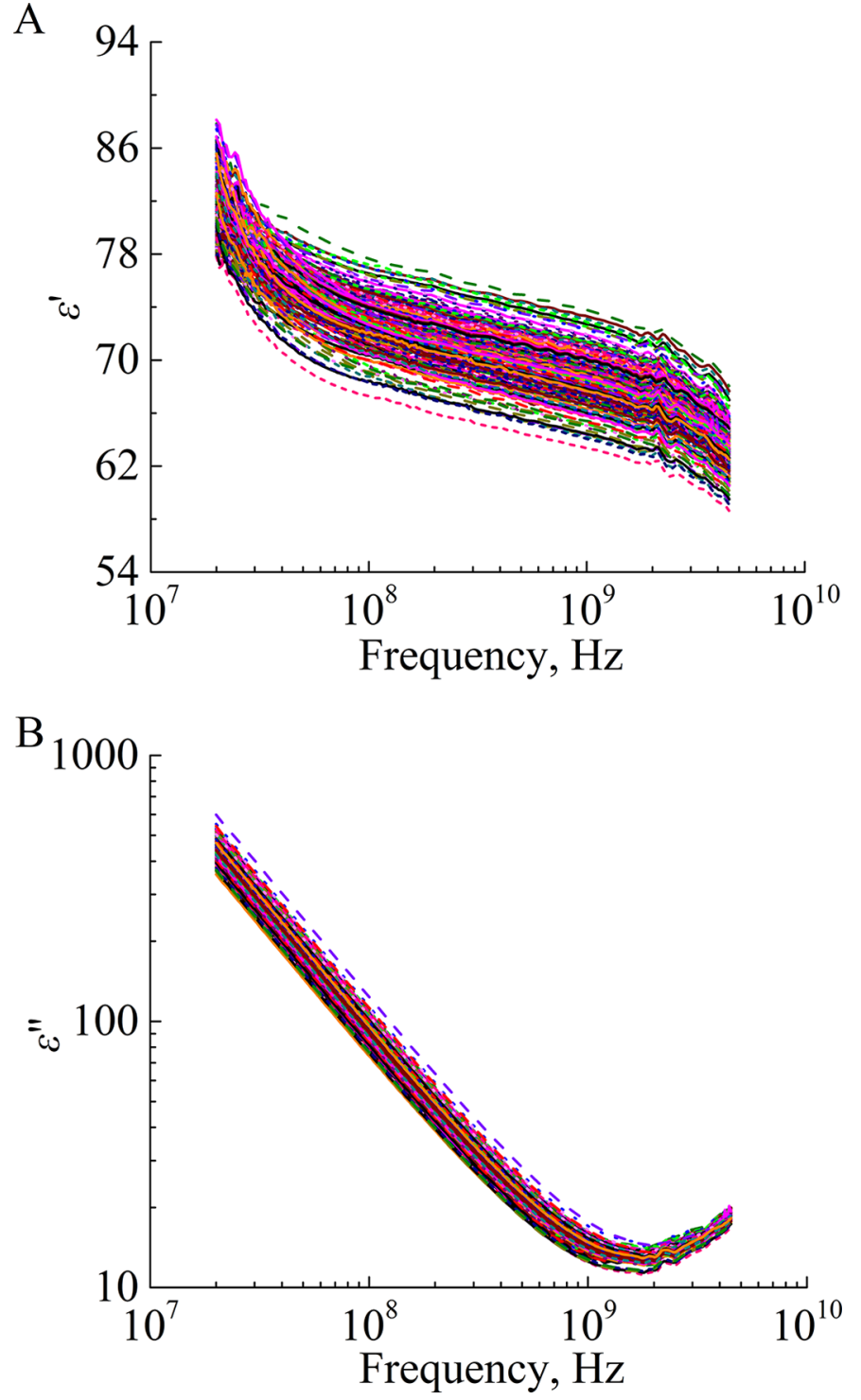

Figure 1. Original dielectric constant $\varepsilon^{\prime}(\mathrm{A})$ and dielectric loss factor $\varepsilon^{\prime \prime}(\mathrm{B})$ spectra of 301 raw cow milk samples. used as the optimal spectral pretreated method, and the 296 samples without outliers were divided into 3:1 using the SPXY method. Finally, the calibration and prediction sets had 222 and 74 samples, respectively. The SCC of the samples in the calibration set was between $3.70 \log \mathrm{SCC} / \mathrm{mL}$ and $6.70 \log \mathrm{SCC} / \mathrm{mL}$ with the standard deviation of $0.63 \log \mathrm{SCC} / \mathrm{mL}$. The $\mathrm{SCC}$ of the samples in the prediction set changed from 4.95 $\log \mathrm{SCC} / \mathrm{mL}$ to $6.65 \log \mathrm{SCC} / \mathrm{mL}$ with the standard deviation of $0.45 \log \mathrm{SCC} / \mathrm{mL}$. The SCC range of the calibration set was wider than that of the prediction set, indicating the samples were divided reasonably.

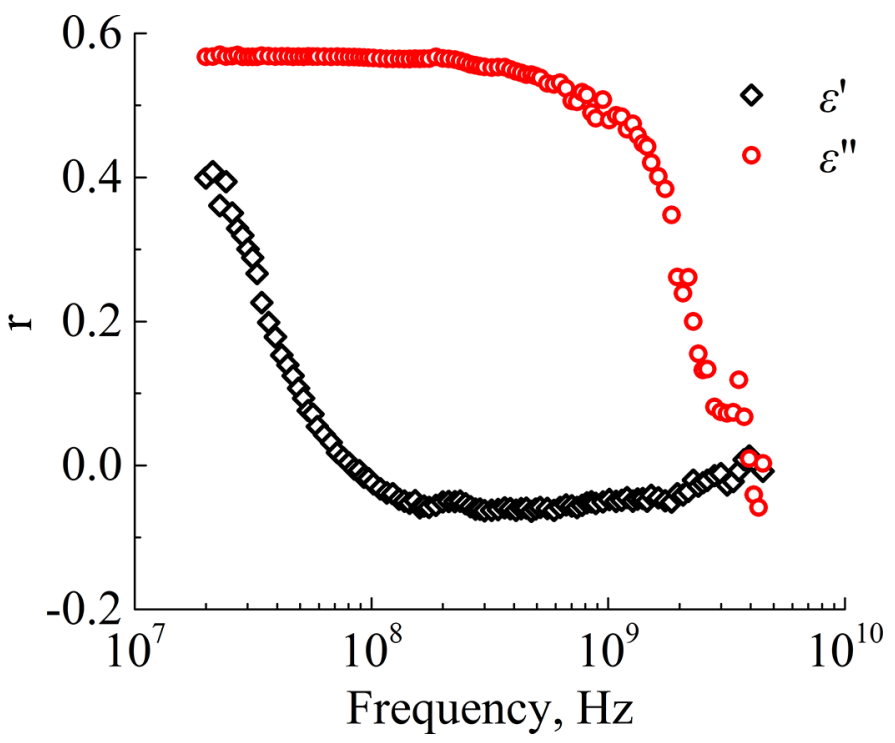

Figure 2. The linear correlation coefficients (r) of SCC with dielectric constant $\varepsilon^{\prime}$ and dielectric loss factor $\varepsilon^{\prime \prime}$ at 201 frequencies from 20 to $4,500 \mathrm{MHz}$ 


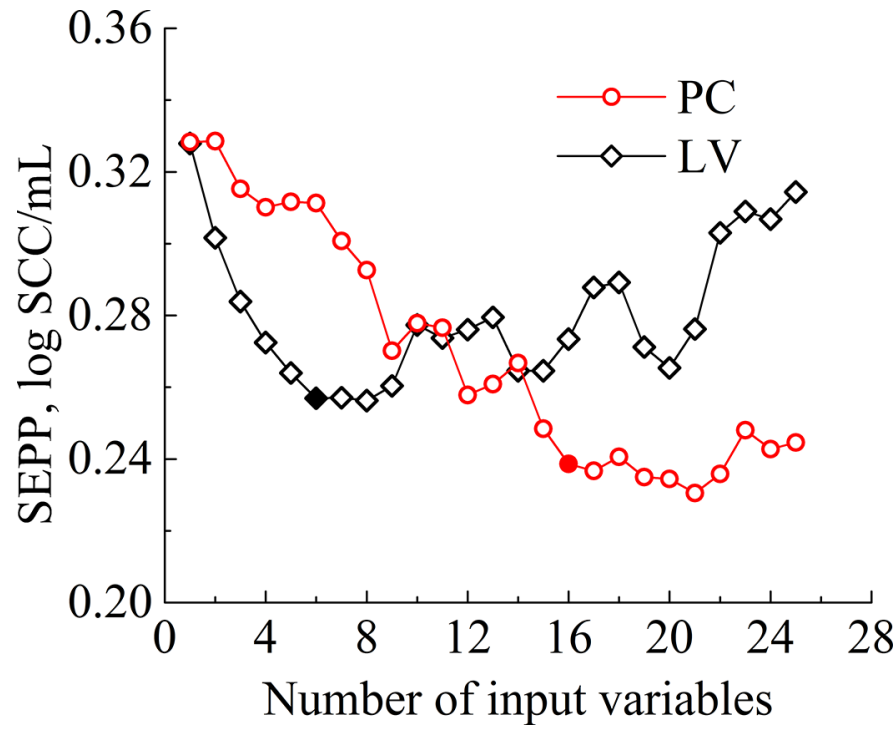

Figure 3. The prediction results of linear support vector regression models for the samples in prediction set based on different numbers of principal components $(\mathrm{PC})$ and latent variables (LV).

\section{Data Reduction}

Figure 3 shows the calculated SEPP of established LSVR models when the numbers of LV and PC increased from 1 to 25 with an interval of 1 . It tells that when the numbers of LV and PC were 8 and 21 , the LSVR models had the smallest SEPP of $0.26 \mathrm{log}$ $\mathrm{SCC} / \mathrm{mL}$ and $0.23 \log \mathrm{SCC} / \mathrm{mL}$, respectively. However, when the numbers of $\mathrm{LV}$ and PC increased from 6 to 8 and from 16 to 21, respectively, there were no obvious changes in the SEPP of LSVR models. Therefore, the optimal numbers of $\mathrm{LV}$ and $\mathrm{PC}$ were decided as 6 and 16 , respectively.

The PLSR models were established when the LV increased from 1 to 25 with an increment of 1 by using the leave-one-out cross-validation method. The optimal PLSR model had the smallest RMSECV of $0.46 \mathrm{log}$ $\mathrm{SCC} / \mathrm{mL}$ with $18 \mathrm{LV}$. When the CVR were selected

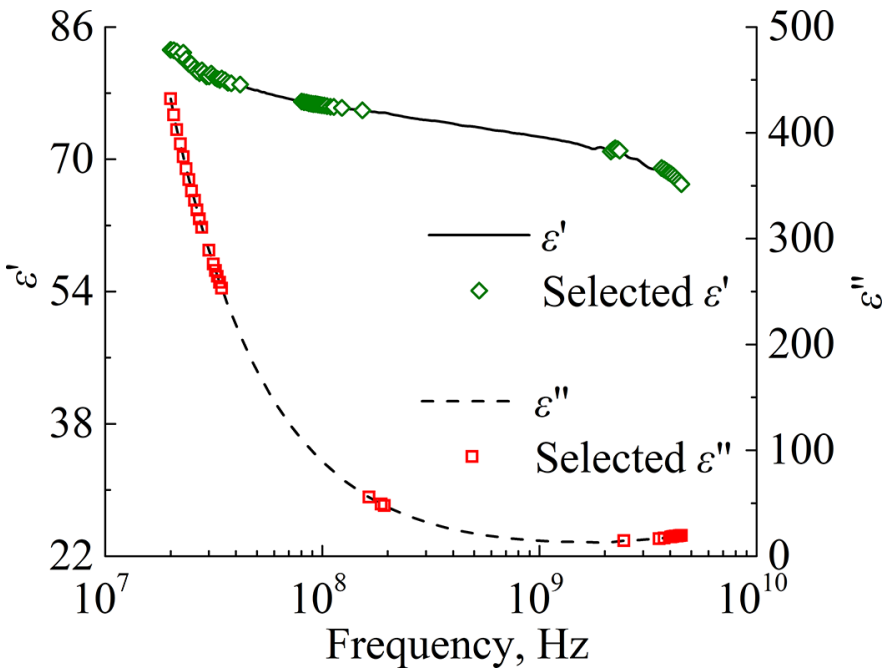

Figure 4. The characteristic variables selected by using variable importance in projection. $\varepsilon^{\prime}=$ dielectric constant; $\varepsilon^{\prime \prime}=$ dielectric loss factor.

based on VIP values, the VIP values of 402 dielectric variables were calculated and between 0.74 and 2.99 . When the threshold was set as 1.0, 86 CVR, including $56 \varepsilon^{\prime}$ and $30 \varepsilon^{\prime \prime}$ variables, were determined and shown in Figure 4.

When selecting CVR using MC-UVE, because the stability of $95 \%$ random variables was less than 3.09 , the stability threshold was determined as 3.09. Finally, 126 variables, including $60 \varepsilon^{\prime}$ and $66 \varepsilon^{\prime \prime}$ variables, were selected as CVR using MC-UVE.

Table 3 lists the prediction results of the LSVR models based on the full spectra (FS), the data after reducing dimension using PLS and PCA, and selected CVR using VIP and MC-UVE. The smallest SEPP, $0.22 \mathrm{log}$ $\mathrm{SCC} / \mathrm{mL}$, was found for the built LSVR models with the selected CVR using VIP and with the FS, followed by $0.24 \log \mathrm{SCC} / \mathrm{mL}$ of the established LSVR models using PCA and selected CVR using MC-UVE. Compared with MC-UVE, the data dimension after data re-

Table 3. The prediction results of linear support vector regression models based on full spectra (FS) and different data reduction methods

\begin{tabular}{lccc}
\hline $\begin{array}{l}\text { Data reduction } \\
\text { method }^{1}\end{array}$ & Data dimension & SEPC $^{2}(\log \mathrm{SCC} / \mathrm{mL})$ & SEPP $^{3}(\log \mathrm{SCC} / \mathrm{mL})$ \\
\hline FS & 402 & 0.44 & 0.22 \\
PLS & 6 & 0.47 & 0.26 \\
PCA & 16 & 0.49 & 0.24 \\
VIP & 86 & 0.45 & 0.22 \\
MC-UVE & 126 & 0.45 & 0.24 \\
\hline
\end{tabular}

${ }^{1} \mathrm{PLS}=$ partial least squares; $\mathrm{PCA}=$ principal component analysis; $\mathrm{VIP}=$ variable importance in projection; MC-UVE = Monte Carlo uninformative variable elimination.

${ }^{2} \mathrm{SEPC}=$ standard error of prediction for calibration

${ }^{3} \mathrm{SEPP}=$ standard error of prediction for prediction. 
Table 4. The SCC prediction results of the linear support vector regression (LSVR), nonlinear support vector regression (NSVR), and nonlinear $\mathrm{Nu}$-Support vector regression (NNuSVR) models based on full spectra (FS) and 2 data reduction methods

\begin{tabular}{|c|c|c|c|c|c|c|}
\hline \multirow{2}{*}{$\begin{array}{l}\text { Modeling } \\
\text { method }\end{array}$} & \multirow[b]{2}{*}{ Data reduction method ${ }^{1}$} & \multicolumn{2}{|r|}{ Calibration set $^{2}$} & \multicolumn{2}{|r|}{ Prediction set ${ }^{3}$} & \multirow[b]{2}{*}{$\mathrm{RPD}^{4}$} \\
\hline & & $\mathrm{r}_{\mathrm{C}}$ & SEPC $(\log \mathrm{SCC} / \mathrm{mL})$ & $\mathrm{r}_{\mathrm{p}}$ & SEPP $(\log \mathrm{SCC} / \mathrm{mL})$ & \\
\hline \multirow{2}{*}{$\overline{L S V R}$} & PCA & 0.60 & 0.49 & 0.85 & 0.24 & 1.89 \\
\hline & VIP & 0.67 & 0.45 & 0.87 & 0.22 & 2.05 \\
\hline \multirow[t]{2}{*}{ NSVR } & FS & 0.86 & 0.32 & 0.91 & 0.19 & 2.37 \\
\hline & PCA & 0.88 & 0.29 & 0.86 & 0.23 & 2.00 \\
\hline \multirow{2}{*}{ NNuSVR } & $\mathrm{PCA}$ & 0.83 & 0.35 & 0.88 & 0.21 & 2.17 \\
\hline & VIP & 0.84 & 0.33 & 0.90 & 0.20 & 2.25 \\
\hline
\end{tabular}

${ }^{1} \mathrm{PCA}=$ principal component analysis; VIP $=$ variable importance in projection.

${ }^{2} \mathrm{r}_{\mathrm{C}}=$ correlation coefficient for calibration; SEPC = standard error of prediction for calibration.

${ }^{3} \mathrm{r}_{\mathrm{p}}=$ correlation coefficient for prediction; SEPP $=$ standard error of prediction for prediction.

${ }^{4} \mathrm{RPD}=$ residual prediction deviation.

duction using PCA was obviously less. Therefore, VIP and PCA had excellent data reduction performance in this study and were used in subsequent modeling.

\section{Modeling Results}

LSVR Modeling Results. Table 4 shows the SCC prediction results of the built LSVR models in the calibration and prediction sets when FS, PCA, and VIP were used as data reduction methods. The SEPP of the built LSVR models with FS (FS-LSVR) and VIP (VIP-LSVR) were $0.22 \log \mathrm{SCC} / \mathrm{mL}$, lower than 0.24 $\log \mathrm{SCC} / \mathrm{mL}$ of the LSVR model based on PCA. Moreover, the $r_{p}$ of FS-LSVR and VIP-LSVR were 0.87. Therefore, among the 3 LSVR models, the FS-LSVR and VIP-LSVR had the best prediction performance for SCC.

NSVR Modeling Results. Combining 5-fold crossvalidation and grid search methods, the regularization coefficient $c$ and RBF width parameter $g$ were optimized to make a minimum RMSECV. The ranges of $c$ and $g$ were set to $2^{-8}$ to $2^{8}$ with an interval of $2^{0.5}$. In this study, the best $c$ and $g$ of NSVR models based on FS (FS-NSVR), PCA (PCA-NSVR), and VIP (VIP-NSVR) were 128.0 and 0.0039, 2.0 and 0.0884, and 128.0 and 0.0039 , respectively.

The SCC prediction results of NSVR models based on FS and different data reduction methods at the optimal values of $c$ and $g$ are listed in Table 4 . The FS-NSVR and VIP-NSVR had the same SEPP of $0.19 \log$ SCC/ $\mathrm{mL}$, lower than $0.23 \log \mathrm{SCC} / \mathrm{mL}$ of the PCA-NSVR. The $r_{p}$ (0.91) of FS-NSVR was higher than that of VIP-NSVR (0.90) and PCA-NSVR (0.86). Therefore, FS-NSVR was regarded as the best model among the NSVR models.
NNuSVR Modeling Results. In addition to $c$ and $g$, a new parameter $v$ was added in the NNuSVR model. The 5-fold cross-validation and grid search methods were also used to decide the optimal combination of $c$, $g$, and $v$ based on the lowest RMSECV. The ranges of $c$ and $g$ were also set from $2^{-8}$ to $2^{8}$ with an interval of $2^{0.5}$. The range of $v$ was set from 0 to 1 with an interval of 0.1. In this study, the best $c, g$, and $v$ of NNuSVR models based on FS (FS-NNuSVR), PCA (PCANNuSVR), and VIP (VIP-NNuSVR) were 45.2548 , 0.0039 , and $0.4 ; 2.0,0.0442$, and 0.5 ; and 128.0, 0.0078, and 0.6 , respectively.

Table 4 shows the SCC prediction results of the built NNuSVR models using the FS and different data reduction methods under the best combination of parameters $c, g$, and $v$. The SEPP of FS-NNuSVR and VIP-NNuSVR were $0.20 \log \mathrm{SCC} / \mathrm{mL}$, lower than 0.21 $\log \mathrm{SCC} / \mathrm{mL}$ of the PCA-NNuSVR. The $r_{p}$ of VIPNNuSVR, FS-NNuSVR, and PCA-NNuSVR were 0.90, 0.89 , and 0.88 , respectively. Therefore, the prediction performance of VIP-NNuSVR was optimal.

\section{Comparison}

We noted that with the same modeling method, VIP was superior to PCA and could provide a prediction performance equal to or exceeding FS. Among different models, PCA always reduced prediction performance when compared with FS. The results indicated that VIP can effectively delete redundant variables, whereas PCA might lose some information related to SCC prediction.

Among 9 established models, the FS-NSVR and VIP-NSVR had the best SCC prediction ability with SEPP and RPD of $0.19 \log \mathrm{SCC} / \mathrm{mL}$ and 2.37, respec- 
tively. The FS-NNuSVR and VIP-NNuSVR also had good SCC prediction performance with SEPP of 0.20 $\log \mathrm{SCC} / \mathrm{mL}$ and RPD of 2.25. Nicolaï et al. (2007) pointed out that the model could make a rough quantitative prediction when the RPD is between 2.0 and 2.5. Therefore, the combination of dielectric spectra and SVR can quantitatively predict the SCC of raw milk.

When compared with the reported prediction performance for the SCC of raw milk using NIR or visible-NIR spectroscopy technology, the obtained lowest SEP $(0.19$ $\log \mathrm{SCC} / \mathrm{mL}$ ) was a little higher than that reported by Melfsen et al. (2012) (0.18 $\log \mathrm{SCC} / \mathrm{mL})$ based on the NIR spectra in 851 to $1,649 \mathrm{~nm}$, but it was lower than the reported SEPP of $0.382 \log \mathrm{SCC} / \mathrm{mL}$ based on the NIR spectra in 1,100 to $2,500 \mathrm{~nm}$ (Tsenkova et al., 2001), the given cross-validation SEP of $0.3 \log$ SCC/ $\mathrm{mL}$ using the shortwave NIR spectra in 700 to $1,100 \mathrm{~nm}$ (Tsenkova et al., 2009), and the obtained SEP of 0.20 $\log \mathrm{SCC} / \mathrm{mL}$ based on the visible-NIR spectra in 700 to 1,050 nm (Iweka et al., 2020). This indicates that the dielectric spectroscopy combined with the SVR model has great potential in predicting the SCC of raw milk.

\section{CONCLUSIONS}

The dielectric spectra measurement system based on the coaxial probe technology and a milk analyzer were used to collect the dielectric spectra and SCC of 301 raw milk samples, respectively. After pretreating original dielectric spectra with SNV, the MD was used for outlier detection. Based on the SPXY algorithm, the samples without outliers were divided into the calibration and prediction sets according to the ratio of 3:1. Dimension reduction (PLS and PCA) and variable selection (VIP and MC-UVE) methods were applied for data reduction. To quantitatively predict the SCC of raw milk, the LSVR, NSVR, and NNuSVR models were established using FS, $16 \mathrm{PC}$ were obtained by PCA, and 86 CVR were extracted by VIP. The optimal models were the FS-NSVR and VIP-NSVR, and their SEPP and RPD were $0.19 \log \mathrm{SCC} / \mathrm{mL}$ and 2.37 , respectively. The results indicated that the combination of dielectric spectra with SVR could realize the simple, fast, and accurate milk SCC detection. This study offers a novel method to determine dairy mastitis based on the dielectric properties of its produced milk.

\section{ACKNOWLEDGMENTS}

The authors gratefully acknowledge the financial support provided by National Natural Science Foundation of China (Project No. 32172308 and No. 31671935; Beijing, China) and Chinese Universities Scientific Fund
(Project No. 2452021159; Yangling, Shaanxi, China). The authors have not stated any conflicts of interest.

\section{REFERENCES}

Ashraf, A., and M. Imran. 2018. Diagnosis of bovine mastitis: From laboratory to farm. Trop. Anim. Health Prod. 50:1193-1202. https://doi.org/10.1007/s11250-018-1629-0.

Blowey, R., and P. Edmondson. 2010. Mastitis control in dairy herds. 2nd ed. CAB International.

Cai, W., Y. Li, and X. Shao. 2008. A variable selection method based on uninformative variable elimination for multivariate calibration of near-infrared spectra. Chemom. Intell. Lab. Syst. 90:188-194. https://doi.org/10.1016/j.chemolab.2007.10.001.

Dalen, G., A. Rachah, H. Norstebo, Y. H. Schukken, and O. Reksen. 2019. The detection of intramammary infections using online somatic cell counts. J. Dairy Sci. 102:5419-5429. https://doi.org/10 $.3168 /$ jds.2018-15295.

Farrés, M., S. Platikanov, S. Tsakovski, and R. Tauler. 2015. Comparison of the variable importance in projection (VIP) and of the selectivity ratio (SR) methods for variable selection and interpretation. J. Chemometr. 29:528-536. https://doi.org/10.1002/cem .2736 .

Galvão, R. K. H., M. C. U. Araujo, G. E. José, M. J. C. Pontes, E. C. Silva, and T. C. B. Saldanha. 2005. A method for calibration and validation subset partitioning. Talanta 67:736-740. https:// doi.org/10.1016/j.talanta.2005.03.025.

Guo, W., X. Zhu, H. Liu, R. Yue, and S. Wang. 2010. Effects of milk concentration and freshness on microwave dielectric properties. J. Food Eng. 99:344-350. https://doi.org/10.1016/j.jfoodeng.2010.03 .015 .

Halasa, T., K. Huijps, O. Østerås, and H. Hogeveen. 2007. Economic effects of bovine mastitis and mastitis management: A review. Vet. Q. 29:18-31. https://doi.org/10.1080/01652176.2007.9695224.

He, W., S. Ma, L. Lei, J. He, X. Li, J. Tao, X. Wang, S. Song, Y. Wang, Y. Wang, J. Shen, C. Cai, and C. Wu. 2020. Prevalence, etiology, and economic impact of clinical mastitis on large dairy farms in China. Vet. Microbiol. 242:108570. https://doi.org/10 .1016/j.vetmic.2019.108570.

Hovinen, M., J. Siivonen, S. Taponen, L. Hänninen, M. Pastell, A. M. Aisla, and S. Pyörälä. 2008. Detection of clinical mastitis with the help of a thermal camera. J. Dairy Sci. 91:4592-4598. https://doi .org/10.3168/jds.2008-1218.

ISO (International Organization for Standardization). 2006. ISO 13366-2: Milk-Enumeration of somatic cells - Part 2: Guidance on the operation of fluoro-opto-electronic counters. ISO.

Iweka, P., S. Kawamura, T. Mitani, T. Kawaguchi, and S. Koseki. 2020. Online milk quality assessment during milking using near-infrared spectroscopic sensing system. Environ. Control Biol. 58:1-6. https://doi.org/10.2525/ecb.58.1.

Kamphuis, C., R. Sherlock, J. Jago, G. Mein, and H. Hogeveen. 2008. Automatic detection of clinical mastitis is improved by in-line monitoring of somatic cell count. J. Dairy Sci. 91:4560-4570. https: //doi.org/10.3168/jds.2008-1160.

Khatun, M., C. E. F. Clark, N. A. Lyons, P. C. Thomson, K. L. Kerrisk, and S. C. García. 2017. Early detection of clinical mastitis from electrical conductivity data in an automatic milking system. Anim. Prod. Sci. 57:1226-1232. https://doi.org/10.1071/AN16707.

Liu, Q., W. Guo, and X. Zhu. 2018. Effect of lactose content on dielectric properties of whole milk and skim milk. Int. J. Food Sci. Technol. 53:2037-2044. https://doi.org/10.1111/ijfs.13790.

Mahalanobis, P. C. 1936. On the generalized distance in statistics. Proceedings of the National Institute of Science of India 2:49-55.

Melfsen, A., E. Hartung, and A. Haeussermann. 2012. Accuracy of in-line milk composition analysis with diffuse reflectance nearinfrared spectroscopy. J. Dairy Sci. 95:6465-6476. https://doi.org/ 10.3168/jds.2012-5388.

Nicolaï, B. M., K. Beullens, E. Bobelyn, A. Peirs, W. Saeys, K. I. Theron, and J. Lammertyn. 2007. Nondestructive measurement 
of fruit and vegetable quality by means of NIR spectroscopy: A review. Postharvest Biol. Technol. 46:99-118. https://doi.org/10 $.1016 /$ j.postharvbio.2007.06.024.

Ogola, H., A. Shitandi, and J. Nanua. 2007. Effect of mastitis on raw milk compositional quality. J. Vet. Sci. 8:237-242. https://doi.org/ 10.4142/jvs.2007.8.3.237.

Patel, P. M., A. Bhat, and G. H. Markx. 2008. A comparative study of cell death using electrical capacitance measurements and dielectrophoresis. Enzyme Microb. Technol. 43:523-530. https://doi.org/10 .1016/j.enzmictec.2008.09.006

Pedregosa, F., G. Varoquaux, A. Gramfort, V. Michel, B. Thirion, O. Grisel, M. Blondel, G. Louppe, P. Prettenhofer, R. Weiss, R. J. Weiss, J. VanderPlas, A. Passos, D. Cournapeau, M. Brucher, M. Perrot, and E. Duchesnay. 2011. Scikit-learn: Machine learning in Python. J. Mach. Learn. Res. 12:2825-2830.

Schölkopf, B., A. J. Smola, R. C. Williamson, and P. L. Bartlett. 2000. New support vector algorithms. Neural Comput. 12:1207-1245. https://doi.org/10.1162/089976600300015565.

Stangaferro, M. L., R. Wijma, L. Caixeta, M. Al Abri, and J. Giordano. 2016. Use of rumination and activity monitoring for the identification of dairy cows with health disorders: Part II. Mastitis. J. Dairy Sci. 99:7411-7421. https://doi.org/10.3168/jds.2016-10908.

Tsenkova, R., S. Atanassova, S. Kawano, and K. Toyoda. 2001. Somatic cell count determination in cow's milk by near-infrared spectroscopy: A new diagnostic tool. J. Anim. Sci. 79:2550-2557. https: //doi.org/10.2527/2001.79102550x.

Tsenkova, R., H. Meilina, S. Kuroki, and D. H. Burns. 2009. Near infrared spectroscopy using short wavelengths and leave-one- cowout cross-validation for quantification of somatic cells in milk. J. Near Infrared Spectrosc. 17:345-351. https://doi.org/10.1255/ jnirs.868.
Xiong, Z.-Q., M.-J. Guo, Y.-X. Guo, J. Chu, Y.-P. Zhuang, and S.L. Zhang. 2008. Real-time viable-cell mass monitoring in highcell-density fed-batch glutathione fermentation by Saccharomyces cerevisiae T65 in industrial complex medium. J. Biosci. Bioeng. 105:409-413. https://doi.org/10.1263/jbb.105.409.

Zhang, S., L. Zhou, B. Ling, and S. Wang. 2016. Dielectric properties of peanut kernels associated with microwave and radio frequency drying. Biosyst. Eng. 145:108-117. https://doi.org/10.1016/j .biosystemseng.2016.03.002.

Zhu, X., W. Guo, Y. Jia, and F. Kang. 2015a. Dielectric properties of raw milk as functions of protein content and temperature. Food Bioproc. Tech. 8:670-680. https://doi.org/10.1007/s11947-014 $-1440-5$.

Zhu, X., W. Guo, and Z. Liang. 2015b. Determination of the fat content in cow's milk based on dielectric properties. Food Bioproc. Tech. 8:1485-1494. https://doi.org/10.1007/s11947-015-1508-x.

Zhu, X., Q. Liu, and W. Guo. 2018a. Dielectric properties of raw milk as influenced by frequency, salts, and salt contents. J. Food Process Eng. 41:e12885. https://doi.org/10.1111/jfpe.12885.

Zhu, Z., X. Zhu, F. Kong, and W. Guo. 2018b. A rapid method on identifying disqualified raw goat's milk based on total bacterial count by using dielectric spectra. J. Food Eng. 239:40-51. https:/ /doi.org/10.1016/j.jfoodeng.2018.06.020.

\section{ORCIDS}

Zhuozhuo Zhu ํ https://orcid.org/0000-0001-5564-5832

Xinhua Zhu (1) https://orcid.org/0000-0001-9576-7690

Wenchuan Guo ๑ https://orcid.org/0000-0002-0550-772X 